\title{
Plasma Velocities in the Solar Corona and Transition Region
}

\author{
Yakunina G.V., Porfir'eva G.A., and Delone A.B. \\ Sternberg Astronomical Institute, Moscow State University, Universitetskij Prospekt 13, \\ Moscow 119992, Russia, email: yakunina@sai.msu.ru
}

\begin{abstract}
A short compilation of results of spectral observations of nonthermal velocities $\xi$ in a wide range of temperatures in X-ray, EUV, UV and visual wave-lengths has been made. Relationship between the $\xi$ and electron temperature $\mathrm{T}$ for chromosphere, quiet and active corona, coronal holes $(\mathrm{CHs})$ and flaring plasma is considered.
\end{abstract}

\section{Data and analysis}

Investigations of the directed $\mathrm{V}$ and random $\xi$ velocities in the different layers of the solar atmosphere help us to understand better the energy transport into the corona. Spectral line profiles, forming in the temperature range from $1 \times 10^{4} \mathrm{~K}$ to $3 \times 10^{7} \mathrm{~K}$, contain information of matter velocities in different layers and coronal structures. Literature data on observations, obtained from cosmic stations and on the ground, and results of interferometric observations during several solar eclipces (see Delone, Yakunina \& Porfir'eva (2003b) have been used.

The relationship between the nonthermal velocity and temperature of the emitting plasma is shown in the Figure 1. The $\xi$ values for the chromosphere, transition region and lower corona (symbol $\square$ ) were taken from Chae et al. (1998). In addition the data for the quiet corona (symbol +) were taken from Delone, Yakunina \& Porfir'eva (2003b), Doschek \& Feldman (1977), and Raju et al. (2000). We can see that the nonthermal velocity increases when the temperature increases, reaching its maximum equal to $\sim 30$ $\mathrm{km} \mathrm{s}^{-1}$ when $\mathrm{T}=3 \times 10^{5} \mathrm{~K}$, and then begins to fall to the value of about $20 \mathrm{~km} \mathrm{~s}^{-1}$ in the lower corona. The $\xi$ values for coronal holes $($ symbol $\times$ ) were taken from Doschek \& Feldman (1977), Hassler \& Moran (1994), Wilhelm et al. (1998), Raju et al. (2000), and Delone et al. (2003a). As it is discussed by Delone et al. (2003a) the random velocities in $\mathrm{CHs}$ in average are greater than in quiet corona. The values of $\xi$ for nonflaring active regions $(A R s)($ symbol $\triangle$ ), being in a relatively quiet station, were taken from Delone, Yakunina \& Porfir'eva (2003b), Saba \& Strong (1991), Sterling (1997), and Harra, Matthews \& Culhane (2001). We can see that the nonthermal velocities and temperature in the ARs are greater than in the lower quiet corona. For the flaring corona the data were taken from Delone, Yakunina \& Porfir'eva (2003b) (symbol o), Harra, Matthews \& Culhane (2001), Ranns et al. (2001), and Doschek, Mariska \& Strong (1994) (symbol •).

As it was shown in Delone, Yakunina \& Porfir'eva (2003b), and Porfir'eva \& Yakunina (2003) the $\bar{\xi}$ for the $\mathrm{X}$-ray flares with $\mathrm{T} \geqslant 10^{7} \mathrm{~K}$ is equal to $(161 \pm 5) \mathrm{km} \mathrm{s}^{-1}$ but the values of $\xi$ vary in the wide range from 90 to $300 \mathrm{~km} \mathrm{~s}^{-1}$. For the weaker flares with $\mathrm{T} \leqslant 10^{7} \mathrm{~K}$ $\bar{\xi}=(146 \pm 8) \mathrm{km} \mathrm{s}^{-1}($ symbol $\diamond)$, i.e. a little less than for the hot flares. The dynamical loops have greater $\xi$ than the quiet ones have (symbols $\mathbf{\square}$ and correspondingly, Chae et al. (2000)). The nonthermal velocities in the coronal holes are greater than in the surrounding quiet coronal regions, and the $\xi$ values in the nonflaring active regions are greater than in the quiet diffuse corona. 


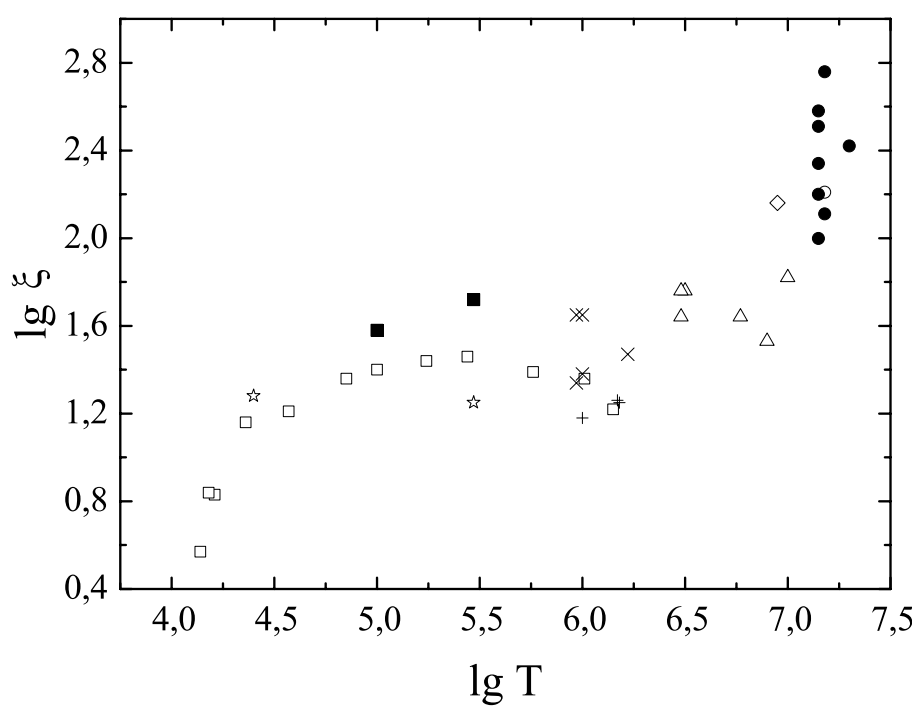

Figure 1. The nonthermal velocities $\xi$ as a function of the electron temperature T.

\section{Summary}

In general the nonthermal velocity increases from several $\mathrm{km} \mathrm{s}^{-1}$ to several hundreds $\mathrm{km} \mathrm{s}^{-1}$ with temperature increase from $10^{4} \mathrm{~K}$ to $3 \times 10^{7} \mathrm{~K}$. But there is no one-toone dependence between the nonthermal velocity and temperature. Different attentions are made to explane the excess nonthermal broadening of the line profiles. The most probable mechanism appears to be due to MHD turbulence although other expanations are possible.

\section{References}

Chae, J., Schuhle, U., \& Lemaire P. 1998 Ap. J. 505, 957-973.

Chae, J., Wang, H., Goode, P. R., \& Wilhelm, K. 2000 Ap. J. 533, 535-545.

Delone, A. B., Porfir'eva, G. A., Smirnova, O. B., \& Yakunina, G. V. 2003a In Internat.Conf. on Solar Physics. 7-11 July 2003. Pulkovo. The climatic and ecological aspects of the solar activity. (eds. V. M. Makarov, Yu. A. Nagovitsyn, and V. N. Obridko), 135-139, (Russian).

Delone, A. B., Yakunina, G. V. \& Porfir'eva, G. A. 2003b Izvestiya Russian Academii Nauk 67, No 3, 381-389.

Doschek, G. A. \& Feldman, U. 1977 Ap. J. 212, L143-L146.

Doschek, G. A., Mariska, J. T. \& Strong, K. T. 1994 Ap. J. 431, 888-900.

Doschek, G. A., Warren, H. P., Laming, J. T., Mariska, J. T., Wilhelm, K., Lemaire, P., Schuhle, U., \& Moran, T. G. 1997 Ap. J. 482, L109-L112.

Harra, L. K., Matthews, S. A. \& Culhane, I. L. 2001 Ap. J. 549, L245-L248.

Hassler, D. M. \& Moran, T. G. 1994 Spase Sci. Rev. 70, 373-377.

Porfir'eva, G. A. \& Yakunina, G. V. 2003 Astronom. and Astrophys. Transactions 22, Nos 4-5, 611-616.

Raju, K. P., Sakurai, T., Ichimoto, K., \& Sing, J. 2000 Ap. J. 543, 1044-1050.

Ranns, N. D. R., Harra, L. K., Matthews, S. A. \& Culhane, I. L. 2001 A \& A 379, 616-621.

Saba, J. L. R. \& Strong, K. T. 1991 Ap. J. 375, 789-799.

Sterling, A. C. 1997 Ap. J. 375, 807-813.

Wilhelm, K., Marsch, E., Dwivedi, B. N., Hassler, D. M., Lemaire, P., Gabriel, A. H., \& Huber, M. C. 1998 Ap. J. 500, 1023-1038. 


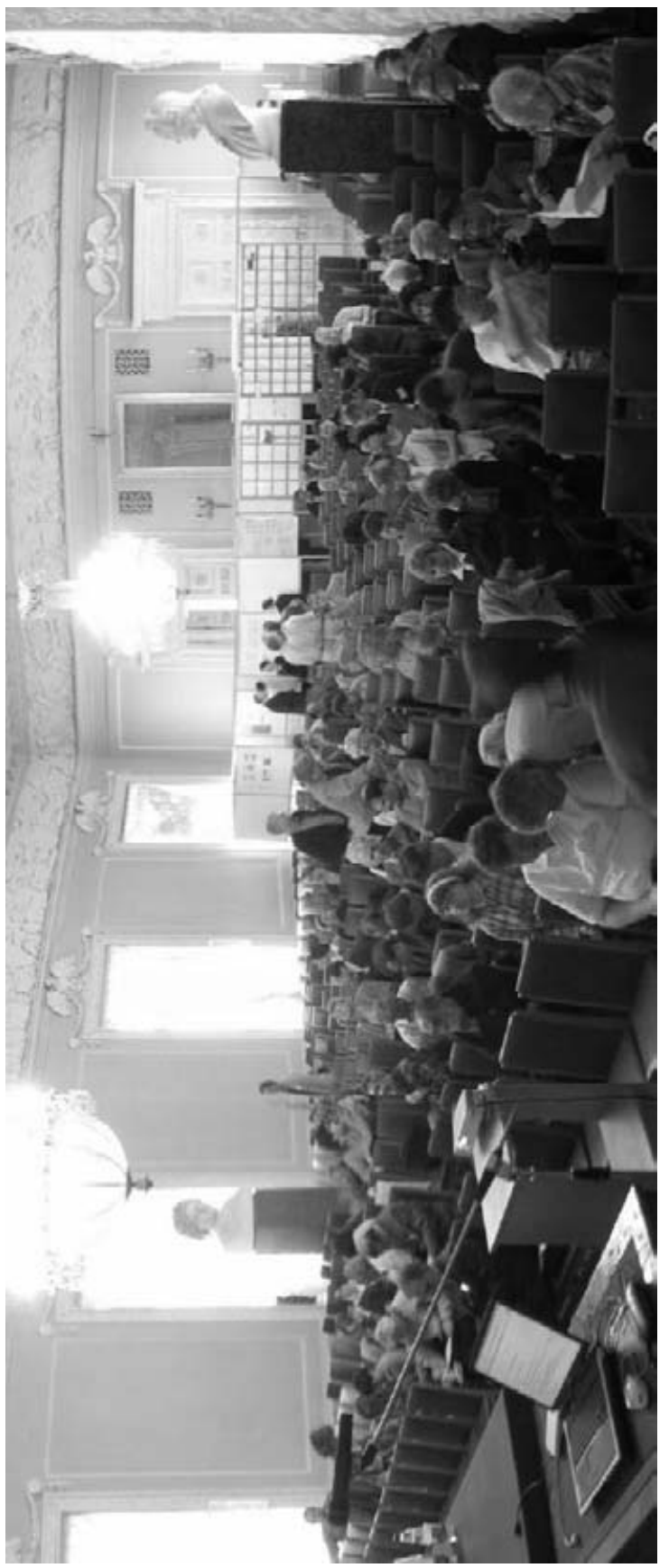

Before the morning session on Monday, June 14, 2004 


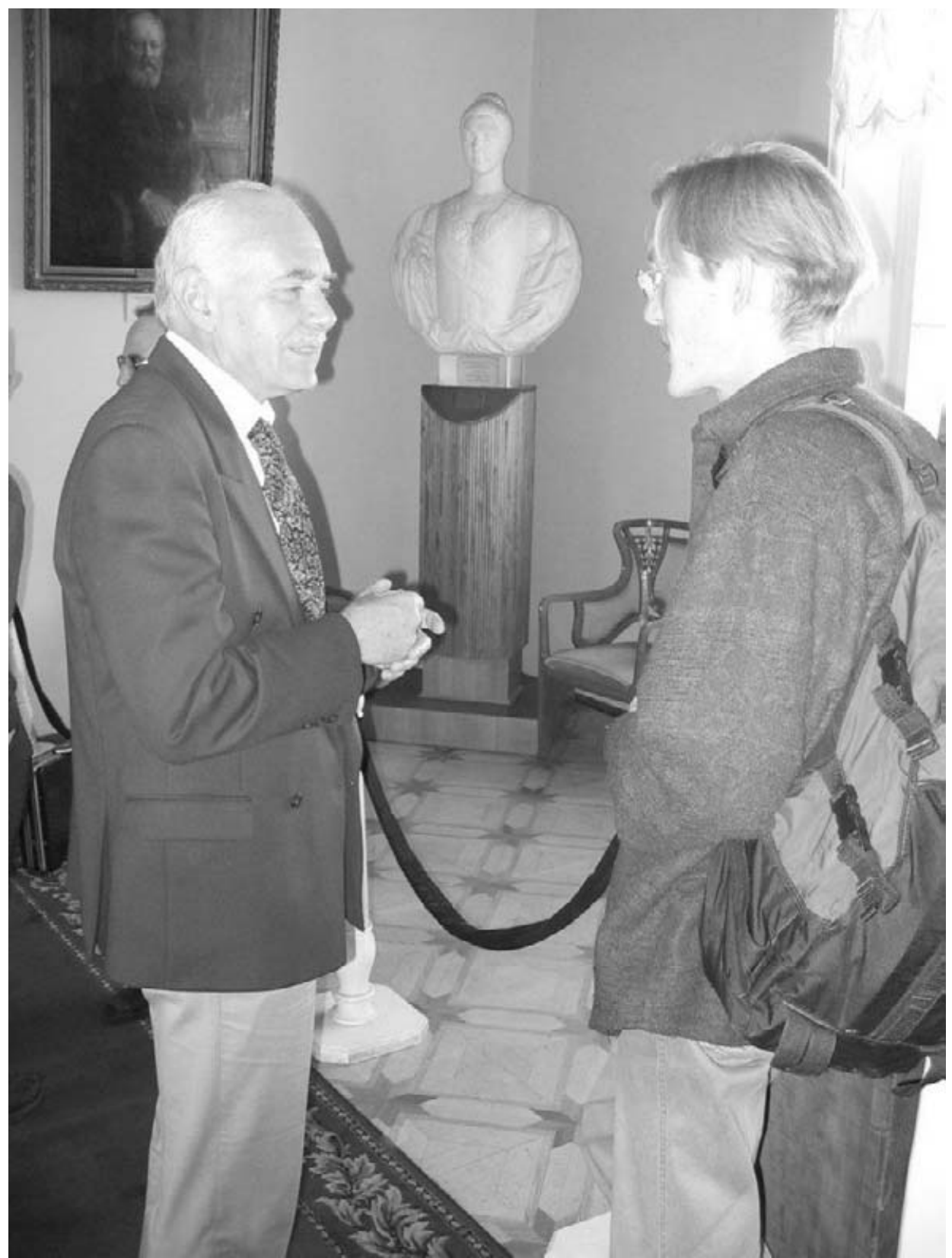

Serge Koutchmy (France). Discussion. 